\title{
Energy Conservation in the Thin Layer Approximation: III. The Spherical Relativistic Case for Supernovae
}

\author{
Lorenzo Zaninetti \\ Physics Department, Turin, Italy \\ Email: zaninetti@ph.unito.it
}

How to cite this paper: Zaninetti, L. (2020) Energy Conservation in the Thin Layer Approximation: III. The Spherical Relativistic Case for Supernovae. International Journal of Astronomy and Astrophysics, 10, 285-301.

https://doi.org/10.4236/ijaa.2020.104015

Received: August 19, 2020

Accepted: October 16, 2020

Published: October 19, 2020

Copyright $\odot 2020$ by author(s) and Scientific Research Publishing Inc. This work is licensed under the Creative Commons Attribution International License (CC BY 4.0).

http://creativecommons.org/licenses/by/4.0/

\begin{abstract}
The theory of the conservation of energy in the thin layer approximation has been extended to special relativity. Four models for the density of the circumstellar medium are analyzed, which are represented by constant, power law, exponential and Emden $(n=5)$ profile for density. The astrophysical results are presented in a numerical way, except for a Taylor expansion of the four trajectories in the surrounding of the origin. The free parameters of the models are particularized for $\mathrm{SN1993j}$, for which the radius versus time is known. Some evaluations on the time dilation are presented.
\end{abstract}

\section{Keywords}

Supernovae: General, Supernovae: Individual (SN1993j), ISM: Supernova Remnants

\section{(c) (i) Open Access}

\section{Introduction}

The production of relativistic electrons in the early phase of a supernova (SN) is an active field of research. For example, the application of the non-relativistic perpendicular shocks to: 1) the formation of Weibel-type filamentation instabilities, which generate magnetic turbulence, see [1];2) the shock-surfing acceleration of electrons at the leading edge of the shock foot and downstream of the shock, see [2] [3]; and 3) studying the magnetic re-connection as a dominant acceleration process for the acceleration of the electrons, see [4]. These approaches use non-relativistic shocks. Therefore, an approach in special relativity (SR) is required. In this paper, we report some approaches to solve this problem, including: the relativistic theory of hydrodynamical shocks, see [5]; the self-similar spherical solution describing an adiabatic ultra relativistic blast wave, see [6]; 
analysis of the reverse shock in a dynamical evolution of a relativistic explosion, see [7]; evaluation of the jump conditions in parallel relativistic collision-less shocks in the absence of Fermi acceleration, see [8]; the ultra-relativistic shock breakout with production of non-thermal emission, which was investigated by [9]; and, an analytic description of relativistic radiation-mediated shocks with application to SN, see [10]. The astronomical measures of the high-velocity features in optical spectra of type Ia supernovae reveal high velocity, $V$, for the ejecta such as $24,000 \mathrm{~km} / \mathrm{s}$, which means $\beta=0.08$; where $\beta=\frac{v}{c}$ with $c$ is the light velocity, see [11]. The measured high velocities in young SNs require a treatment for the early expansion in the framework of SR. Previous studies analyzed the relativistic conservation of momentum for the thin layer approximation adopting a power law profile of the density, see [12], and a Lane Emden ( $n$ =5) profile of density, see [13]. We recall that the relativistic conservation of the momentum or the energy in the thin layer approximation is a hypothesis of work that should be sustained from the observations, i.e. the observed trajectory of SN 1993J [14]. This paper is structured as follows. In Section 2, the basic equations of the conservation of the relativistic energy for the thin layer approximation are described. In Section 3, the astrophysical results for SN 1993J for four density profiles of the circumstellar medium (CSM) are given. Finally, time dilation and radioactivity are outlined in Section 4.

\section{The Relativistic Framework}

\subsection{Energy Conservation}

The classical conservation of kinetic energy in spherical coordinates within the framework of the thin layer approximation when the thermal effects are negligible is

$$
\frac{1}{2} M_{0}\left(r_{0}\right) v_{0}^{2}=\frac{1}{2} M(r) v^{2},
$$

where $M_{0}\left(r_{0}\right)$ and $M(r)$ are the swept masses at $r_{0}$ and $r$, and $v_{0}$ and $v$ are the velocities of the thin layer at $r_{0}$ and $r$, for further details, see [15].

In $\mathrm{SR}$, the total energy of a particle is

$$
E=M \gamma c^{2}
$$

where $M$ is the rest mass, $c$ is the light velocity, $\gamma$ is the Lorentz factor $\frac{1}{\sqrt{1-\beta^{2}}}, \quad \beta=\frac{v}{c}$ and $v$ the velocity. The relativistic kinetic energy, $E_{k}$, is

$$
E_{k}=M c^{2}(\gamma-1)
$$

where the rest energy has been subtracted from the total energy, see formula (23.1) in [16]. The relativistic conservation of kinetic energy in the thin layer approximation in two points $\left(r_{0}, v_{0}\right)$ and $(r, v)$ is

$$
M_{0}\left(r_{0}\right) c^{2}\left(\gamma_{0}-1\right)=M(r) c^{2}(\gamma-1)
$$


where $M_{0}\left(r_{0}\right)$ and $M(r)$ are the swept masses at $r_{0}$ and $r$, respectively, $\gamma_{0}=\frac{1}{\sqrt{1-\beta_{0}^{2}}}$ and $\beta_{0}=\frac{v_{0}}{c}$. A Taylor expansion about $v=0$ and $v_{0}=0$ of order three for the above relativistic conservation law gives the classic case given by Equation (1). This fact assures a smooth transition from relativistic to classical velocities.

\subsection{Constant Density}

When the ISM has a constant density, the Lorentz factor as function of the radius is

$$
\beta\left(r ; r_{0}, \beta_{0}\right)=\frac{B N}{B D},
$$

where

$$
\begin{gathered}
B N=-r_{0}^{3 / 2}\left[-2\left(\left(\left(r^{3}-\frac{1}{2} r_{0}^{3}\right) \beta_{0}^{2}-r^{3}+r_{0}^{3}\right) \sqrt{C}+\left(-r^{3}+r_{0}^{3}\right) \beta_{0}^{2}+r^{3}-r_{0}^{3}\right)\right. \\
\times\left(\left(\left(r^{6}-2 r^{3} r_{0}^{3}+r_{0}^{6}\right) \beta_{0}^{2}-r^{6}+2 r^{3} r_{0}^{3}-2 r_{0}^{6}\right) \sqrt{C}\right. \\
\left.\left.+\left(2 r^{3} r_{0}^{3}-2 r_{0}^{6}\right) \beta_{0}^{2}-2 r^{3} r_{0}^{3}+2 r_{0}^{6}\right)\right)^{\frac{1}{2}}, \\
B D=\sqrt{C} \beta_{0}^{2} r^{6}-2 \sqrt{C} \beta_{0}^{2} r^{3} r_{0}^{3}+\sqrt{C} \beta_{0}^{2} r_{0}^{6}+2 \beta_{0}^{2} r^{3} r_{0}^{3}-2 \beta_{0}^{2} r_{0}^{6} \\
\quad-\sqrt{C} r^{6}+2 \sqrt{C} r^{3} r_{0}^{3}-2 \sqrt{C} r_{0}^{6}-2 r^{3} r_{0}^{3}+2 r_{0}^{6},
\end{gathered}
$$

and

$$
C=1-\beta_{0}^{2} .
$$

The differential equation that regulates the motion can be obtained from the above equation by inserting $\frac{\mathrm{d} r}{\mathrm{~d} t}=v=\beta \times c$ and $v_{0}=\beta_{0} \times c$,

$$
\frac{\mathrm{d} r\left(t ; r_{0}, v_{0}, c\right)}{\mathrm{d} t}=\frac{C N}{C D}
$$

where

$$
\begin{aligned}
C N= & r_{0}^{3 / 2} \sqrt{4}\left[\left(\left(\left(-\frac{1}{2} c^{2}+\frac{1}{2} r_{0}^{2}\right)(r(t))^{6}+\left(c^{2} r_{0}^{3}-r_{0}^{5}\right)(r(t))^{3}\right.\right.\right. \\
& \left.\left.-r_{0}^{6}\left(c^{2}-\frac{1}{2} r_{0}^{2}\right)\right) \sqrt{c^{2}-r_{0}^{2}}+\left(-r_{0}^{3} c^{3}+c r_{0}^{5}\right)(r(t))^{3}+r_{0}^{6} c^{3}-c r_{0}^{8}\right) \\
& \times\left(\left(\left(c^{2}-r_{0}^{2}\right)(r(t))^{3}-r_{0}^{3}\left(c^{2}-\frac{1}{2} r_{0}^{2}\right)\right) \sqrt{c^{2}-r_{0}^{2}}\right. \\
& \left.\left.+\left(-c^{3}+c r_{0}^{2}\right)(r(t))^{3}+r_{0}^{3} c^{3}-c r_{0}^{5}\right)\right]^{\frac{1}{2}} c
\end{aligned}
$$

and 


$$
\begin{aligned}
C D= & \sqrt{c^{2}-r_{0}^{2}}(r(t))^{6} c^{2}-\sqrt{c^{2}-r_{0}^{2}}(r(t))^{6} r_{0}^{2}-2 \sqrt{c^{2}-r_{0}^{2}}(r(t))^{3} c^{2} r_{0}^{3} \\
& +2 \sqrt{c^{2}-r_{0}^{2}}(r(t))^{3} r_{0}^{5}+2 \sqrt{c^{2}-r_{0}^{2}} c^{2} r_{0}^{6}-\sqrt{c^{2}-r_{0}^{2}} r_{0}^{8} \\
& +2(r(t))^{3} c^{3} r_{0}^{3}-2(r(t))^{3} c r_{0}^{5}-2 r_{0}^{6} c^{3}+2 c r_{0}^{8} .
\end{aligned}
$$

This differential does not have an analytical solution and therefore the solution should be derived in a numerical way, except about $r=r_{0}$, where a third-order Taylor series expansion gives

$$
\begin{aligned}
r\left(t ; r_{0}, v_{0}, t_{0}\right) & =r_{0}+v_{0}\left(t-t_{0}\right) \\
& +\frac{3}{2} \frac{\left(c-v_{0}\right)\left(c+v_{0}\right)\left(c^{2}-c \sqrt{c^{2}-v_{0}^{2}}-v_{0}^{2}\right)\left(t-t_{0}\right)^{2}}{c \sqrt{c^{2}-v_{0}^{2}} r_{0}} .
\end{aligned}
$$

\subsection{A Power Law Profile for the Density}

The medium is supposed to scale as

$$
\rho\left(r ; r_{0}\right)= \begin{cases}\rho_{c} & \text { if } r \leq r_{0} \\ \rho_{c}\left(\frac{r_{0}}{r}\right)^{\alpha} & \text { if } r>r_{0}\end{cases}
$$

where $\rho_{c}$ is the density at $r=0, r_{0}$ is the radius after which the density starts to decrease and $\alpha>0$.

The total mass swept, $M\left(r ; r_{0}, \rho_{c}, \alpha\right)$, in the interval $[0, \mathrm{r}]$ is

$$
M\left(r ; r_{0}, \rho_{c}, \alpha\right)=\frac{4}{3} \rho_{c} \pi r_{0}^{3}-4 \frac{r^{3} \rho_{c} \pi}{\alpha-3}\left(\frac{r_{0}}{r}\right)^{\alpha}+4 \frac{\rho_{c} \pi r_{0}^{3}}{\alpha-3} .
$$

The conservation of energy in SR gives the following differential equation

$$
\frac{\mathrm{d} r\left(t ; r_{0}, v_{0}, c\right)}{\mathrm{d} t}=\frac{P N}{P D},
$$

where

$$
\begin{aligned}
P N= & -r_{0}^{3 / 2} c\left[-54\left(\left(1 / 9\left(\left(\alpha^{2}-18 \alpha+72\right) c^{2}+\left(\alpha^{2}+6 \alpha-54\right) v_{0}^{2}\right) r_{0}^{6+\alpha}(r(t))^{-\alpha+3}\right.\right.\right. \\
& +2 / 3(\alpha-15 / 2) r_{0}^{2 \alpha+3}\left(c+v_{0}\right)\left(c-v_{0}\right)(r(t))^{-2 \alpha+6}+r_{0}^{3 \alpha}\left(c^{2}-v_{0}^{2}\right)(r(t))^{-3 \alpha+9} \\
& \left.-1 / 9\left(\left(\alpha^{2}-18\right) v_{0}^{2}+(\alpha-6)^{2} c^{2}\right) r_{0}^{9}\right)\left(c+v_{0}\right)\left(c-v_{0}\right) c \sqrt{\left(c^{2}-v_{0}^{2}\right)^{-1}} \\
& -1 / 9\left(\left(\alpha^{2}-18 \alpha+72\right) c^{2}-3(6+\alpha) v_{0}^{2}\right)\left(c+v_{0}\right)\left(c-v_{0}\right) r_{0}^{6+\alpha}(r(t))^{-\alpha+3} \\
& -2 / 3 r_{2}^{2 \alpha+3}\left((\alpha-15 / 2) c^{2}+1 / 4(\alpha+15) v_{0}^{2}\right)\left(c+v_{0}\right)\left(c-v_{0}\right)(r(t))^{-2 \alpha+6} \\
& -r_{0}^{3 \alpha}\left(c-v_{0}\right)^{2}\left(c+v_{0}\right)^{2}(r(t))^{-3 \alpha+9}+1 / 9 r_{0}^{9}\left((\alpha-6)^{2} c^{4}\right. \\
& \left.\left.\left.-1 / 6\left(\alpha^{3}-3 \alpha^{2}-36 \alpha+216\right) v_{0}^{2} c^{2}+3 / 2(\alpha+3) v_{0}^{4}\right)\right)(\alpha-3)\right]^{\frac{1}{2}}
\end{aligned}
$$

and 


$$
\begin{aligned}
P D= & -6 \sqrt{\frac{c^{2}}{c^{2}-v_{0}^{2}}} \alpha c^{2} r_{0}^{6}+6 \sqrt{\frac{c^{2}}{c^{2}-v_{0}^{2}}} \alpha r_{0}^{6} v_{0}^{2}-\alpha^{2} c^{2} r_{0}^{6}+18 \sqrt{\frac{c^{2}}{c^{2}-v_{0}^{2}}} c^{2} r_{0}^{6} \\
& -18 \sqrt{\frac{c^{2}}{c^{2}-v_{0}^{2}}} r_{0}^{6} v_{0}^{2}+6 \alpha c^{2} r_{0}^{6}-18 c^{2} r_{0}^{6}+9 r_{0}^{6} v_{0}^{2} \\
& +6 \sqrt{\frac{c^{2}}{c^{2}-v_{0}^{2}}} r_{0}^{\alpha+3}(r(t))^{-\alpha+3} \alpha c^{2}-6 \sqrt{\frac{c^{2}}{c^{2}-v_{0}^{2}}} r_{0}^{\alpha+3}(r(t))^{-\alpha+3} \alpha v_{0}^{2} \\
& -18 \sqrt{\frac{c^{2}}{c^{2}-v_{0}^{2}}} r_{0}^{\alpha+3}(r(t))^{-\alpha+3} c^{2}+18 \sqrt{\frac{c^{2}}{c^{2}-v_{0}^{2}}} r_{0}^{\alpha+3}(r(t))^{-\alpha+3} v_{0}^{2} \\
& +18 r_{0}^{\alpha+3}(r(t))^{-\alpha+3} c^{2}-18 r_{0}^{\alpha+3}(r(t))^{-\alpha+3} v_{0}^{2} \\
& -9 r_{0}^{2 \alpha}(r(t))^{-2 \alpha+6} c^{2}+9 r_{0}^{2 \alpha}(r(t))^{-2 \alpha+6} v_{0}^{2} .
\end{aligned}
$$

A third-order Taylor series expansion gives

$$
r\left(t ; t_{0}, r_{0}, v_{0}\right)=r_{0}+v_{0}\left(t-t_{0}\right)-3 / 2 \frac{\left(c-v_{0}\right)\left(c+v_{0}\right)\left(c-\sqrt{c^{2}-v_{0}^{2}}\right)\left(t-t_{0}\right)^{2}}{r_{0} c} .
$$

\subsection{An Exponential Profile}

We assume that the medium around the $\mathrm{SN}$ scales with the piecewise dependence

$$
\rho\left(r ; r_{0}\right)= \begin{cases}\rho_{c} & \text { if } r \leq r_{0} \\ \rho_{c}\left(\exp -\frac{r}{b}\right) & \text { if } r>r_{0}\end{cases}
$$

where $\rho_{c}$ is the density at $r=0$ and $r_{0}$ is the radius after which the density starts to decrease. The total mass swept, $M\left(r ; r_{0}, \rho_{c}\right)$, in the interval $[0, r]$ is

$$
\begin{aligned}
M\left(r ; r_{0}, \rho_{c}, b\right)= & \frac{4}{3} \rho_{c} \pi r_{0}^{3}-4 b\left(2 b^{2}+2 b r+r^{2}\right) \rho_{c} \mathrm{e}^{-\frac{r}{b}} \pi \\
& +4 b\left(2 b^{2}+2 b r_{0}+r_{0}^{2}\right) \rho_{c} \mathrm{e}^{-\frac{r_{0}}{b}} \pi .
\end{aligned}
$$

The conservation of energy in SR gives the following differential equation

$$
\frac{\mathrm{d} r\left(t ; r_{0}, v_{0}, c, b\right)}{\mathrm{d} t}=\frac{E N}{E D},
$$

where

$$
\begin{aligned}
E N= & \mathrm{e}^{\frac{3}{2} \frac{r(t)+r_{0}}{b}} r_{0}^{\frac{3}{2}}\left[\left(\mathrm{e}^{\frac{r(t)+r_{0}}{b}} r_{0}^{3} v_{0}^{2}+12\left(\left(-b^{2}-b r_{0}-\frac{1}{2} r_{0}^{2}\right) \mathrm{e}^{\frac{r(t)}{b}}\right.\right.\right. \\
& \left.\left.+\mathrm{e}^{\frac{r_{0}}{b}}\left(b^{2}+b r(t)+\frac{1}{2}(r(t))^{2}\right)\right) b\left(v_{0}+c\right)\left(-v_{0}+c\right)\right) \sqrt{c^{2}-v_{0}^{2}} \\
& -12\left(\left(-b^{2}-b r_{0}-\frac{1}{2} r_{0}^{2}\right) \mathrm{e}^{\frac{r(t)}{b}}+\mathrm{e}^{\frac{r_{0}}{b}}\left(b^{2}+b r(t)+\frac{1}{2}(r(t))^{2}\right)\right) \\
& \left.\times b\left(v_{0}+c\right)\left(-v_{0}+c\right) c\right]^{\frac{1}{2}}\left[\left(\mathrm{e}^{\frac{2 r(t)+2 r_{0}}{b}} c^{2} r_{0}^{6}-72 b^{2}\left(v_{0}+c\right)\right.\right.
\end{aligned}
$$




$$
\begin{aligned}
& \times\left(-v_{0}+c\right)\left(\left(b^{2}+b r_{0}+\frac{1}{2} r_{0}^{2}\right)\left(b^{2}+b r(t)+\frac{1}{2}(r(t))^{2}\right) \mathrm{e}^{\frac{r(t)+r_{0}}{b}}\right. \\
& \left.\left.-\frac{1}{2} \mathrm{e}^{2 \frac{r(t)}{b}}\left(b^{2}+b r_{0}+\frac{1}{2} r_{0}^{2}\right)^{2}-\frac{1}{2}\left(b^{2}+b r(t)+\frac{1}{2}(r(t))^{2}\right)^{2} \mathrm{e}^{2 \frac{r_{0}}{b}}\right)\right) \sqrt{c^{2}-v_{0}^{2}} \\
& -12 b\left(v_{0}+c\right) r_{0}^{3}\left(-v_{0}+c\right) c\left(\left(b^{2}+b r(t)+\frac{1}{2}(r(t))^{2}\right) \mathrm{e}^{\frac{r(t)+2 r_{0}}{b}}\right. \\
& \left.\left.-\mathrm{e}^{\frac{2 r(t)+r_{0}}{b}}\left(b^{2}+b r_{0}+\frac{1}{2} r_{0}^{2}\right)\right)\right]^{\frac{1}{2}}
\end{aligned}
$$

and

$$
\begin{aligned}
E D= & \frac{1}{c}\left[\left(-72\left(-v_{0}+c\right)\left(b^{2}+b r_{0}+\frac{1}{2} r_{0}^{2}\right)\left(v_{0}+c\right) b^{2}\left(b^{2}+b r(t)+\frac{1}{2}(r(t))^{2}\right)\right.\right. \\
& \times \mathrm{e}^{\frac{2 r_{0}+2 r(t)}{b}}+\mathrm{e}^{\frac{3 r_{0}+3 r(t)}{b}} c^{2} r_{0}^{6}+36\left(-v_{0}+c\right)\left(\mathrm{e}^{\frac{3 r_{0}+r(t)}{b}\left(b^{2}+b r(t)+\frac{1}{2}(r(t))^{2}\right)^{2}}\right. \\
& \left.\left.+\left(b^{2}+b r_{0}+\frac{1}{2} r_{0}^{2}\right)^{2} \mathrm{e}^{\frac{3 r(t)+r_{0}}{b}}\right)\left(v_{0}+c\right) b^{2}\right) \sqrt{c^{2}-v_{0}^{2}}-12 r_{0}^{3}\left(-v_{0}+c\right)\left(v_{0}+c\right) \\
& \left.\times b\left(\left(-b^{2}-b r_{0}-\frac{1}{2} r_{0}^{2}\right) \mathrm{e}^{\frac{2 r_{0}+3 r(t)}{b}}+\mathrm{e}^{\frac{3 r_{0}+2 r(t)}{b}}\left(b^{2}+b r(t)+\frac{1}{2}(r(t))^{2}\right)\right) c\right] .
\end{aligned}
$$

A third-order Taylor series expansion gives

$$
\begin{aligned}
& r\left(t ; t_{0}, r_{0}, v_{0}, b\right) \\
& =r_{0}+v_{0}\left(t-t_{0}\right)+3 / 2 \frac{\left(-v_{0}+c\right)\left(v_{0}+c\right)\left(c^{2}-c \sqrt{c^{2}-v_{0}^{2}}-v_{0}^{2}\right)\left(t-t_{0}\right)^{2}}{c \sqrt{c^{2}-v_{0}^{2}} r_{0}} \mathrm{e}^{-\frac{r_{0}}{b}} \\
& +\frac{v_{0}\left(v_{0}+c\right)\left(-v_{0}+c\right)\left(t-t_{0}\right)^{3}}{b r^{2}}\left(\left(\sqrt{c^{2}-v_{0}^{2}}-c\right)\left(b-\frac{1}{2} r_{0}\right) c \mathrm{e}^{\frac{r_{0}}{b}}\right. \\
& \left.+6 b\left(c \sqrt{c^{2}-v_{0}^{2}}-c^{2}+3 / 4 v_{0}^{2}\right)\right) \frac{1}{\sqrt{\mathrm{e}^{\frac{r_{0}}{b}}}}
\end{aligned}
$$

\subsection{Emden Profile}

We assume that the medium around the $\mathrm{SN}$ scales as a $n=5$ Emden profile, [13] [17] [18],

$$
\rho\left(r ; r_{0}\right)= \begin{cases}\rho_{c} & \text { if } r \leq r_{0} \\ \rho_{c} \frac{1}{\left(1+\frac{1}{3} \frac{r^{2}}{b^{2}}\right)^{5 / 2}} & \text { if } r>r_{0}\end{cases}
$$

where $\rho_{c}$ is the density at $r=0$ and $b$ is the scale.

The total mass swept, $M\left(r ; r_{0}, \rho_{c}\right)$, in the interval $[0, \mathrm{r}]$ is 


$$
M\left(r ; r_{0}, \rho_{c}, b\right)=\frac{4}{3} \rho_{c} \pi r_{0}^{3}+4 \frac{b^{3} r^{3} \rho_{c} \sqrt{3} \pi}{\left(3 b^{2}+r^{2}\right)^{3 / 2}}-4 \frac{b^{3} r_{0}^{3} \rho_{c} \sqrt{3} \pi}{\left(3 b^{2}+r_{0}^{2}\right)^{3 / 2}} .
$$

The conservation of energy in SR gives the following differential equation

$$
\frac{\mathrm{d} r\left(t ; r_{0}, v_{0}, c, b\right)}{\mathrm{d} t}=\frac{D N}{D D},
$$

where

$$
\begin{aligned}
& D N=243 c r_{0}^{3 / 2}\left[( b ^ { 2 } + \frac { 1 } { 3 } ( r ( t ) ) ^ { 2 } ) \left(\frac { 1 } { 3 } ( v _ { 0 } + c ) r _ { 0 } ^ { 3 } \left((r(t))^{3}\left(b^{2}+\frac{1}{3} r_{0}^{2}\right)^{2}\right.\right.\right. \\
& \left.\times \sqrt{3 b^{2}+(r(t))^{2}}-r_{0}^{3}\left(b^{2}+\frac{1}{3}(r(t))^{2}\right)^{2} \sqrt{3 b^{2}+r_{0}^{2}}\right)\left(b^{2}+\frac{1}{3}(r(t))^{2}\right) \\
& \times b^{3}\left(b^{2}+\frac{1}{3} r_{0}^{2}\right) c\left(-v_{0}+c\right) \sqrt{3} \sqrt{\left(c^{2}-v_{0}^{2}\right)^{-1}}-\frac{1}{3}(r(t))^{3}\left(v_{0}+c\right) r_{0}^{3} \\
& \times\left(b^{2}+1 / 3(r(t))^{2}\right) b^{6}\left(b^{2}+\frac{1}{3} r_{0}^{2}\right)\left(-v_{0}+c\right) \sqrt{3 b^{2}+r_{0}^{2}} \sqrt{3 b^{2}+(r(t))^{2}} \\
& +\left(\left(\frac{1}{2} c^{2}-\frac{1}{2} v_{0}^{2}\right) b^{12}+r_{0}^{2}\left(\frac{1}{2} c^{2}-\frac{1}{2} v_{0}^{2}\right) b^{10}+r_{0}^{4}\left(1 / 6 c^{2}-1 / 6 v_{0}^{2}\right) b^{8}\right. \\
& \left.+1 / 18\left(c^{2}-2 / 3 v_{0}^{2}\right) r_{0}^{6} b^{6}+\frac{b^{4} c^{2} r_{0}^{8}}{54}+\frac{b^{2} c^{2} r_{0}^{10}}{162}+\frac{c^{2} r_{0}^{12}}{1458}\right)(r(t))^{6} \\
& +\frac{1}{3}\left(\left(c^{2}-\frac{1}{2} v_{0}^{2}\right) b^{6}+\frac{1}{2} b^{4} c^{2} r_{0}^{2}+1 / 6 b^{2} c^{2} r_{0}^{4}+\frac{c^{2} r_{0}^{6}}{54}\right) r_{0}^{6} b^{2}(r(t))^{4} \\
& +\left(\left(c^{2}-\frac{1}{2} v_{0}^{2}\right) b^{6}+\frac{1}{2} b^{4} c^{2} r_{0}^{2}+1 / 6 b^{2} c^{2} r_{0}^{4}+\frac{c^{2} r_{0}^{6}}{54}\right) r_{0}^{6} b^{4}(r(t))^{2} \\
& \left.+\left(\left(c^{2}-\frac{1}{2} v_{0}^{2}\right) b^{6}+\frac{1}{2} b^{4} c^{2} r_{0}^{2}+1 / 6 b^{2} c^{2} r_{0}^{4}+\frac{c^{2} r_{0}^{6}}{54}\right) r_{0}^{6} b^{6}\right) \\
& \times\left(2 / 3\left(v_{0}+c\right)(r(t))^{3}\left(b^{2}+\frac{1}{3} r_{0}^{2}\right)^{2} \sqrt{3 b^{2}+(r(t))^{2}}\right. \\
& \left.-r_{0}^{3}\left(b^{2}+\frac{1}{3}(r(t))^{2}\right)^{2} \sqrt{3 b^{2}+r_{0}^{2}}\right) b^{3} c\left(-v_{0}+c\right) \sqrt{3} \sqrt{\left(c^{2}-v_{0}^{2}\right)^{-1}} \\
& -2 / 3(r(t))^{3}\left(v_{0}+c\right) b^{3}\left(b^{2}+\frac{1}{3} r_{0}^{2}\right)^{2}\left(-v_{0}+c\right) \sqrt{9 b^{2}+3(r(t))^{2}} \\
& +r_{0}^{3}\left(b^{2}+\frac{1}{3}(r(t))^{2}\right)^{2}\left(2 / 3 b^{3}\left(-v_{0}+c\right)\left(v_{0}+c\right) \sqrt{9 b^{2}+3 r_{0}^{2}}\right. \\
& \left.\left.+\left(b^{2}+\frac{1}{3} r_{0}^{2}\right)^{2} v_{0}^{2}\right)\right)^{\frac{1}{2}} \sqrt{18 b^{2}+6 r_{0}^{2}}
\end{aligned}
$$

and 


$$
\begin{aligned}
D N= & -486\left(v_{0}+c\right) r_{0}^{3}\left[\left((r(t))^{3}\left(b^{2}+\frac{1}{3} r_{0}^{2}\right)^{2} \sqrt{3 b^{2}+(r(t))^{2}}\right.\right. \\
& \left.-r_{0}^{3}\left(b^{2}+\frac{1}{3}(r(t))^{2}\right)^{2} \sqrt{3 b^{2}+r_{0}^{2}}\right)\left(b^{2}+\frac{1}{3}(r(t))^{2}\right) b^{3}\left(b^{2}+\frac{1}{3} r_{0}^{2}\right) \\
& \times c\left(-v_{0}+c\right) \sqrt{3} \sqrt{\left(c^{2}-v_{0}^{2}\right)^{-1}}+486(r(t))^{3}\left(v_{0}+c\right) r_{0}^{3}\left(b^{2}+\frac{1}{3}(r(t))^{2}\right) \\
& \times b^{6}\left(b^{2}+\frac{1}{3} r_{0}^{2}\right)\left(-v_{0}+c\right) \sqrt{3 b^{2}+r_{0}^{2}} \sqrt{3 b^{2}+(r(t))^{2}} \\
& +\left(\left(-729 c^{2}+729 v_{0}^{2}\right) b^{12}+\left(-729 c^{2}+729 v_{0}^{2}\right) r_{0}^{2} b^{10}+\left(-243 c^{2}+243 v_{0}^{2}\right) r_{0}^{4} b^{8}\right. \\
& \left.+\left(-81 c^{2}+54 v_{0}^{2}\right) r_{0}^{6} b^{6}-27 b^{4} c^{2} r_{0}^{8}-9 b^{2} c^{2} r_{0}^{10}-c^{2} r_{0}^{12}\right)(r(t))^{6} \\
& +\left(\left(-486 c^{2}+243 v_{0}^{2}\right) r_{0}^{6} b^{8}-243 b^{6} c^{2} r_{0}^{8}-81 b^{4} c^{2} r_{0}^{10}-9 b^{2} c^{2} r_{0}^{12}\right)(r(t))^{4} \\
& -1458\left(\left(c^{2}-\frac{1}{2} v_{0}^{2}\right) b^{6}+\frac{1}{2} b^{4} c^{2} r_{0}^{2}+1 / 6 b^{2} c^{2} r_{0}^{4}+\frac{c^{2} r_{0}^{6}}{54}\right) r_{0}^{6} b^{4}(r(t))^{2} \\
& \left.-1458\left(\left(c^{2}-\frac{1}{2} v_{0}^{2}\right) b^{6}+\frac{1}{2} b^{4} c^{2} r_{0}^{2}+1 / 6 b^{2} c^{2} r_{0}^{4}+\frac{c^{2} r_{0}^{6}}{54}\right)\right] r_{0}^{6} b^{6} .
\end{aligned}
$$

A third-order Taylor series expansion gives

$$
\begin{aligned}
& r\left(t ; t_{0}, r_{0}, v_{0}, b\right) \\
& =r_{0}+v_{0}\left(t-t_{0}\right)-\frac{\left(-27 v_{0}+27 c\right)\left(v_{0}+c\right)\left(c \sqrt{c^{2}-v_{0}^{2}}-c^{2}+v_{0}^{2}\right) \sqrt{3} b^{5}\left(t-t_{0}\right)^{2}}{2\left(3 b^{2}+r_{0}^{2}\right)^{5 / 2} \sqrt{c^{2}-v_{0}^{2}} r_{0} c} .
\end{aligned}
$$

\section{Astrophysical Observations}

We now analyze in detail the case of SN 1993J; note that the radius in pc and the elapsed time in years can be found in Table 1 of [14].

\subsection{Statistics}

A test for the quality of the fits is represented by the merit function $\chi^{2}$

$$
\chi^{2}=\sum_{j} \frac{\left(r_{t h}-r_{o b s}\right)^{2}}{\sigma_{o b s}^{2}},
$$

where $r_{t h}, r_{o b s}$ and $r_{o b s}$ are the theoretical radius, the observed radius and the observed uncertainty, respectively. A fit can be done by assuming a power law dependence of the type

$$
r(t)=r_{p} t^{\alpha_{p}}
$$

where the two parameters $r_{p}$ and $\alpha_{p}$, as well as their uncertainties can be found using the recipes suggested in [12]. Figure 1 reports the power law fit to the data.

\subsection{Theoretical Fits}

In the case of a constant profile of density, we present a numerical solution as 
given by the numerical integration of the differential Equation (11). Figure 2 displays the theoretical model versus the astronomical data. Figure 3 presents the Taylor approximation of the trajectory as given by (12) in the restricted range of time $[0.001 \mathrm{yr}-0.02 \mathrm{yr}]$.

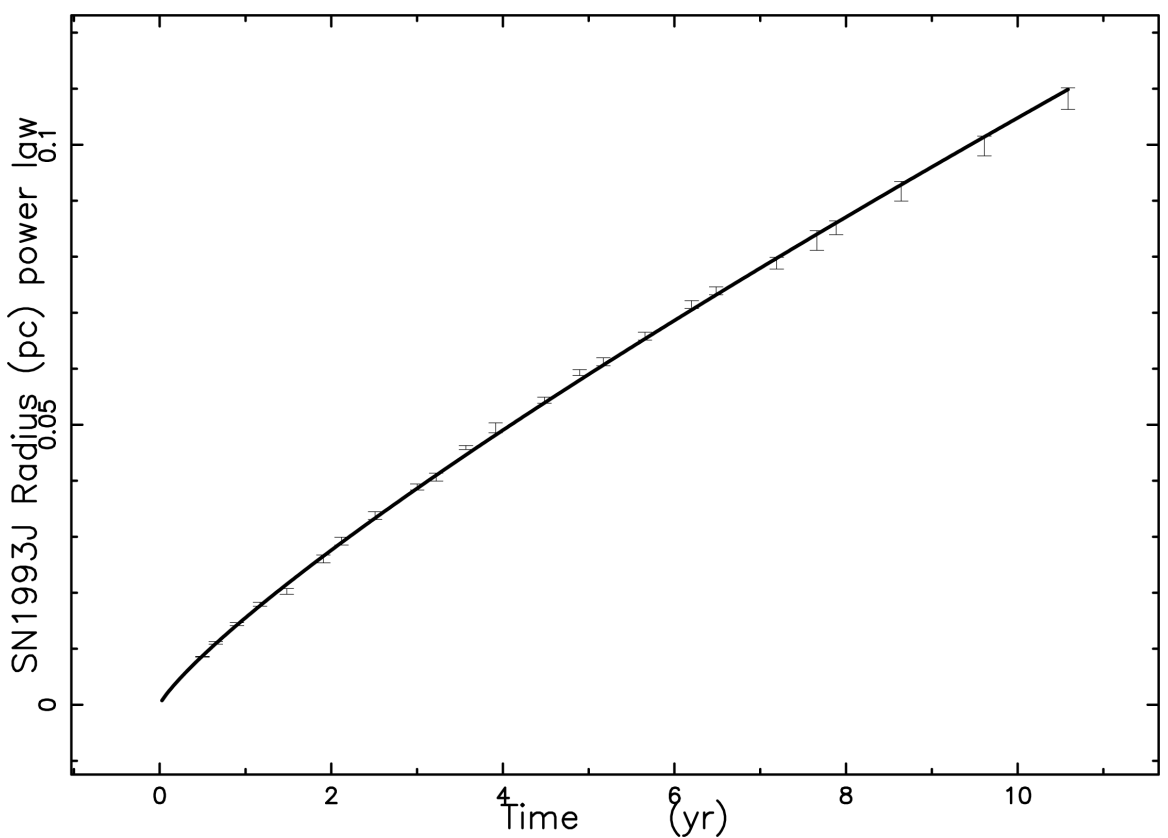

Figure 1. Radius as fitted by a power law (full line) and astronomical data of SN 1993J with vertical error bars. The parameters of the fit are $r_{p}=0.0155 \mathrm{pc}$ and $\alpha_{p}=0.828$, which gives $\chi^{2}=6387$.

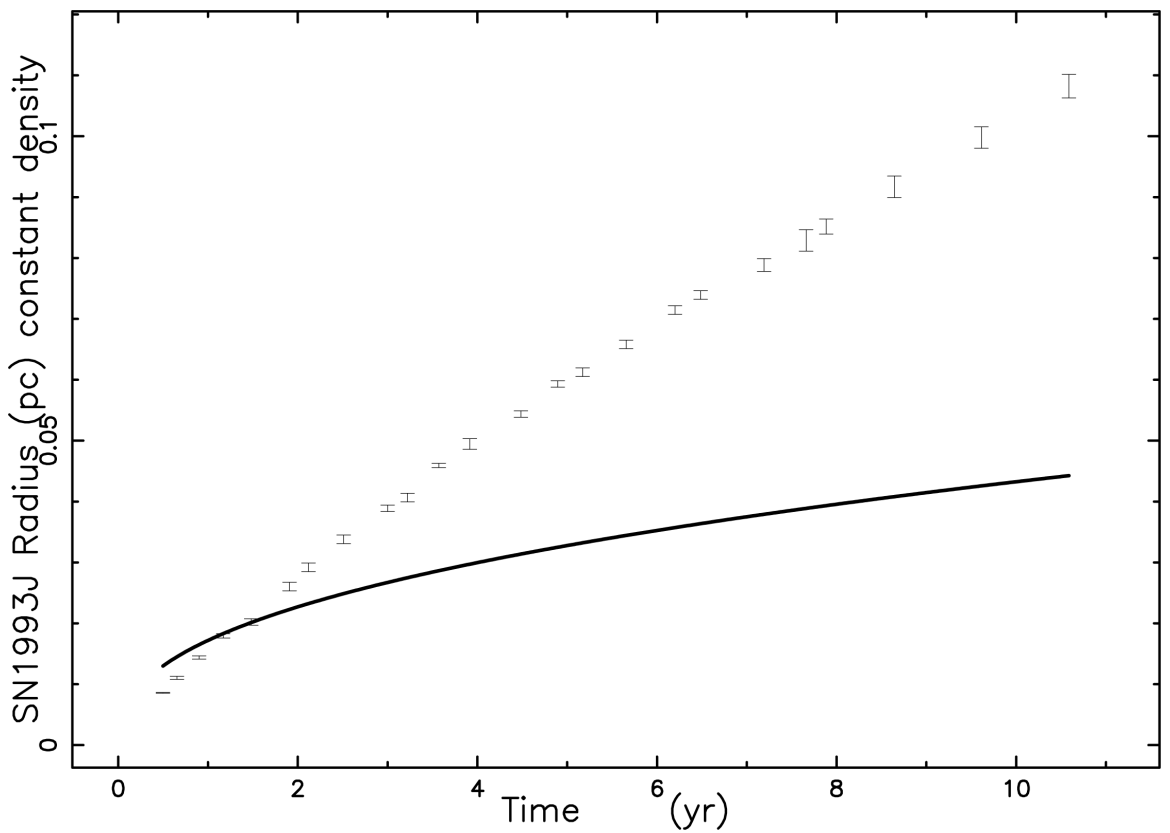

Figure 2. Theoretical radius in the case of constant density (full line) and astronomical data of SN 1993J with vertical error bars. The parameters of the model are $r_{0}=10^{-3} \mathrm{pc}$, $t_{0}=3.6 \times 10^{-3} \mathrm{yr}, \beta_{0}=0.9$ which gives $\chi^{2}=28208$. 
In the case of a power law profile for density, we present a numerical solution as given by the numerical integration of the differential Equation (14). Figure 4 displays the theoretical model.

Figure 5 presents the Taylor approximation of the trajectory as given by (3.2) in the restricted range of time $\left[7.2 \times 10^{-5} \mathrm{yr}-2.2 \times 10^{-4} \mathrm{yr}\right]$.

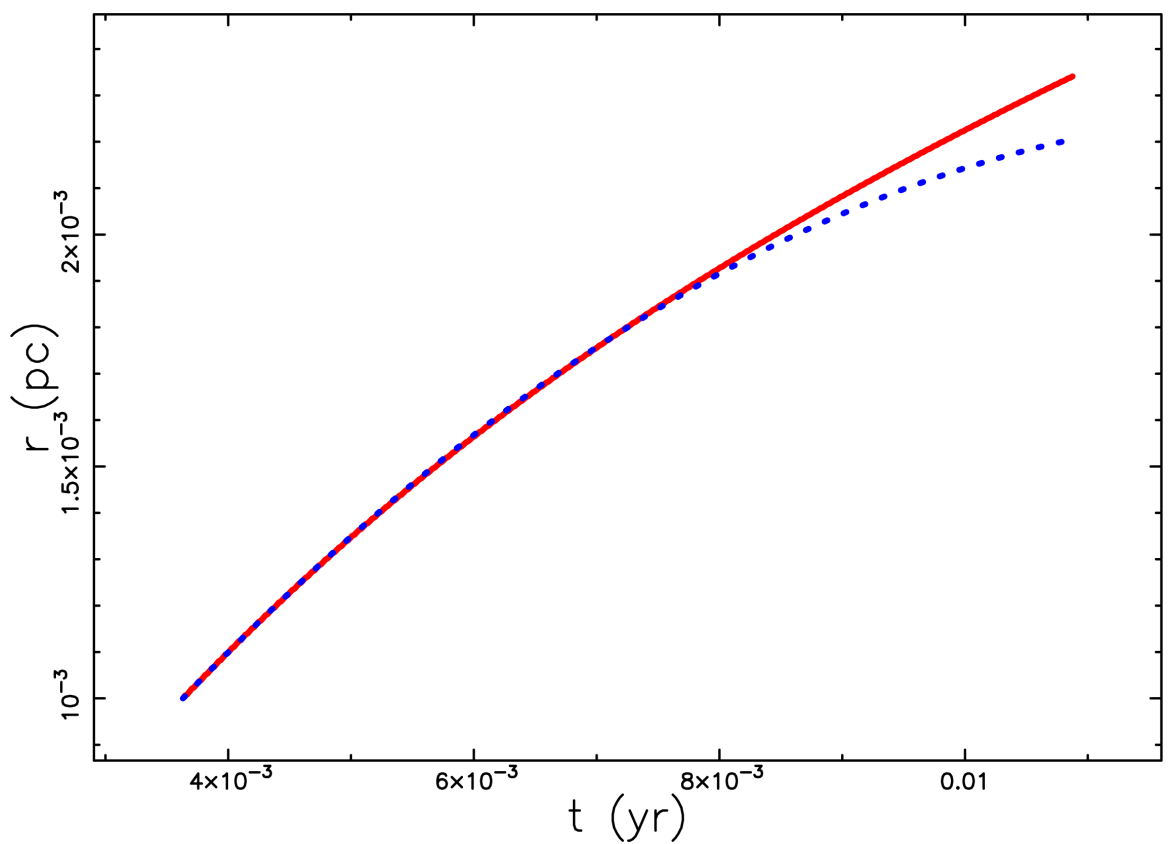

Figure 3. Numerical solution (full-red line) and Taylor solution (blue-dashed line) for the constant density, parameters as Figure 2.

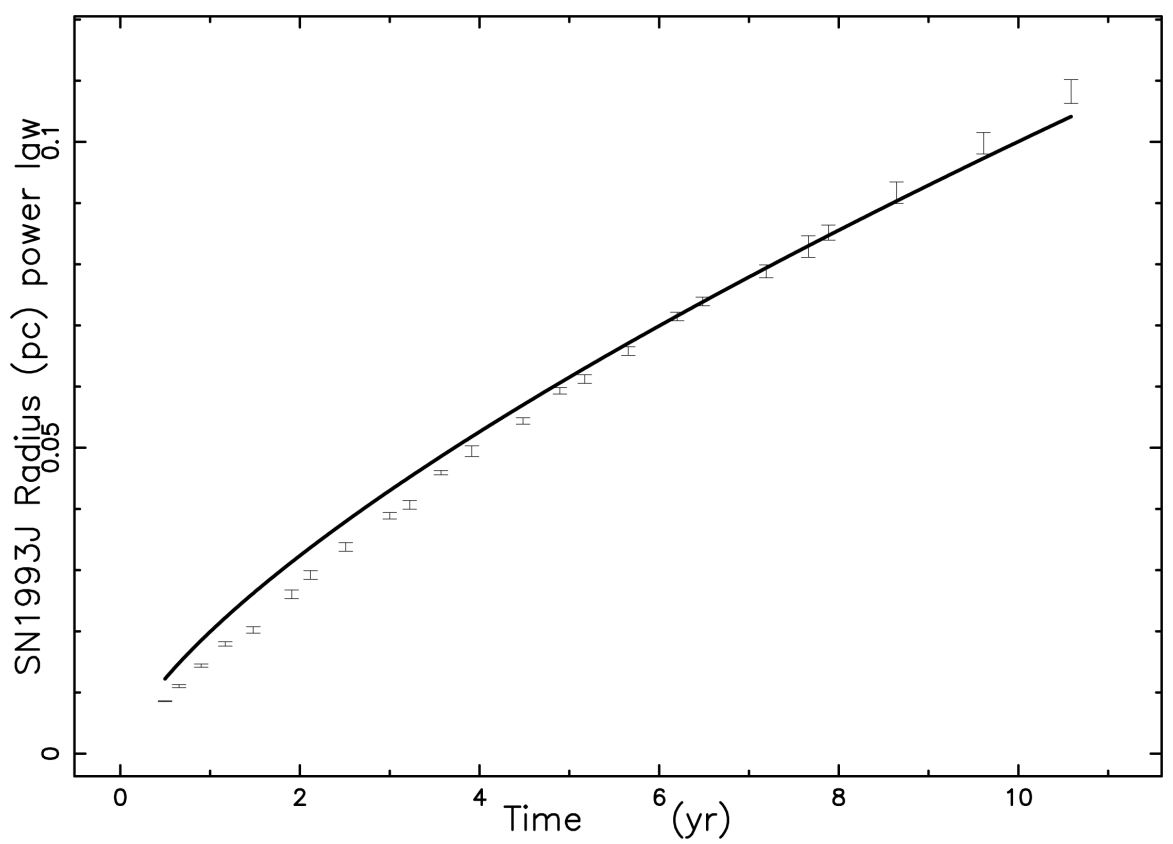

Figure 4. Theoretical radius in the case of a power law profile (full line) and astronomical data of SN 1993J with vertical error bars. The parameters of the model are $r_{0}=2 \times 10^{-5} \mathrm{pc}$, $t_{0}=7.2 \times 10^{-5} \mathrm{yr}, \beta_{0}=0.9, \alpha=2.15$, which gives $\chi^{2}=3777$. 
In the case of an exponential profile for density, we present a numerical solution as given by the numerical integration of the differential Equation (20); Figure 6 displays the theoretical model.

Figure 7 presents the Taylor approximation of the trajectory as given by (23) in the restricted range of time $\left[10^{-3} \mathrm{yr}-8 \times 10^{-3} \mathrm{yr}\right]$.

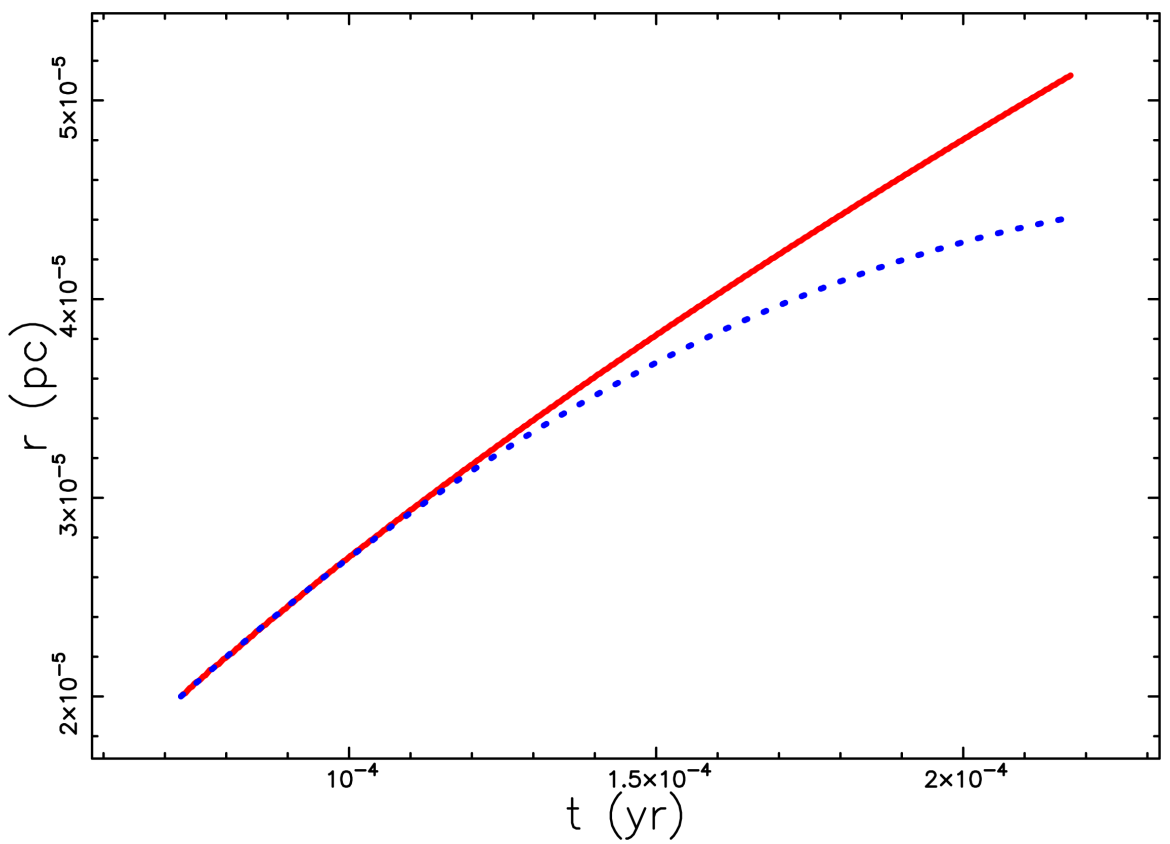

Figure 5. Numerical solution (full-red line) and Taylor solution (blue-dashed line) for the power law profile, the parameters are the same as in Figure 4.

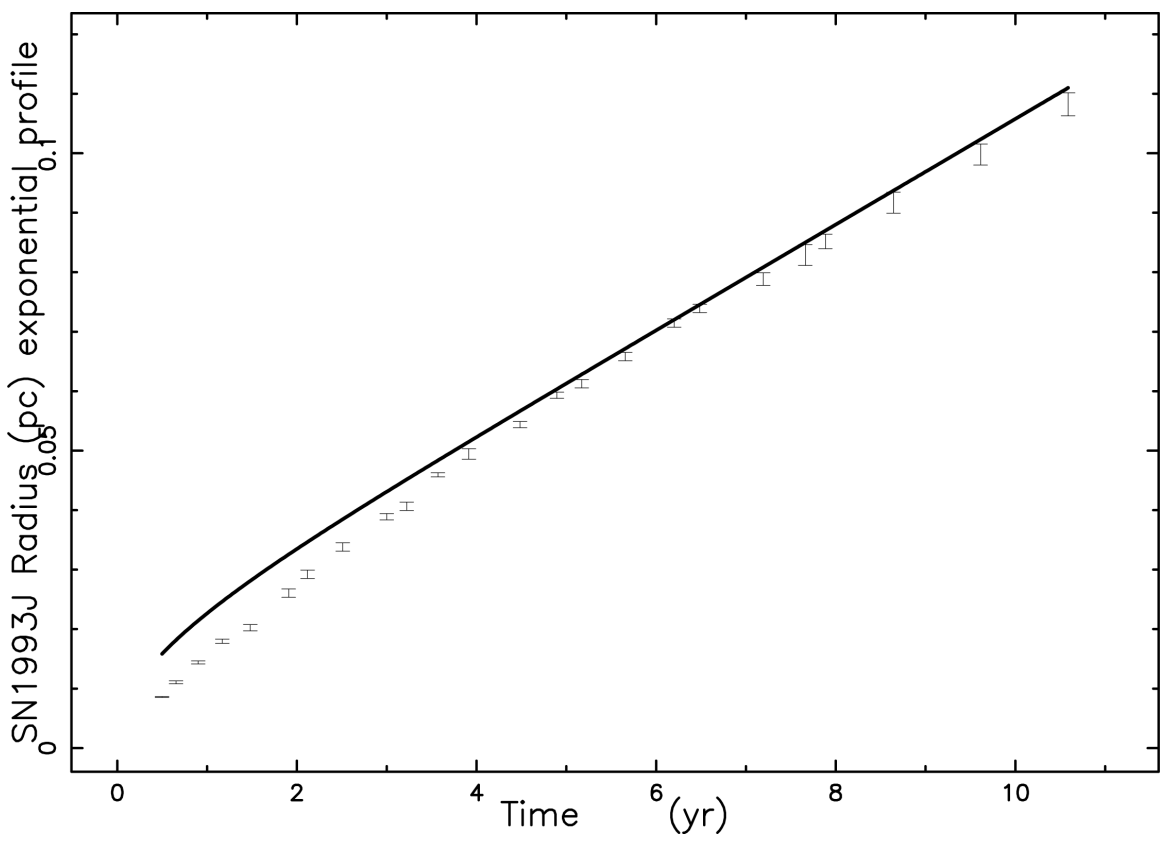

Figure 6. Theoretical radius in the case of an exponential profile (full line) and astronomical data of SN 1993J with vertical error bars. The parameters of the model are $r_{0}=10^{-3} \mathrm{pc}, t_{0}=3.6 \times 10^{-3} \mathrm{yr}, \beta_{0}=0.9$ and $b=8 \times 10^{-3} \mathrm{pc}$ which gives $\chi^{2}=13145$. 


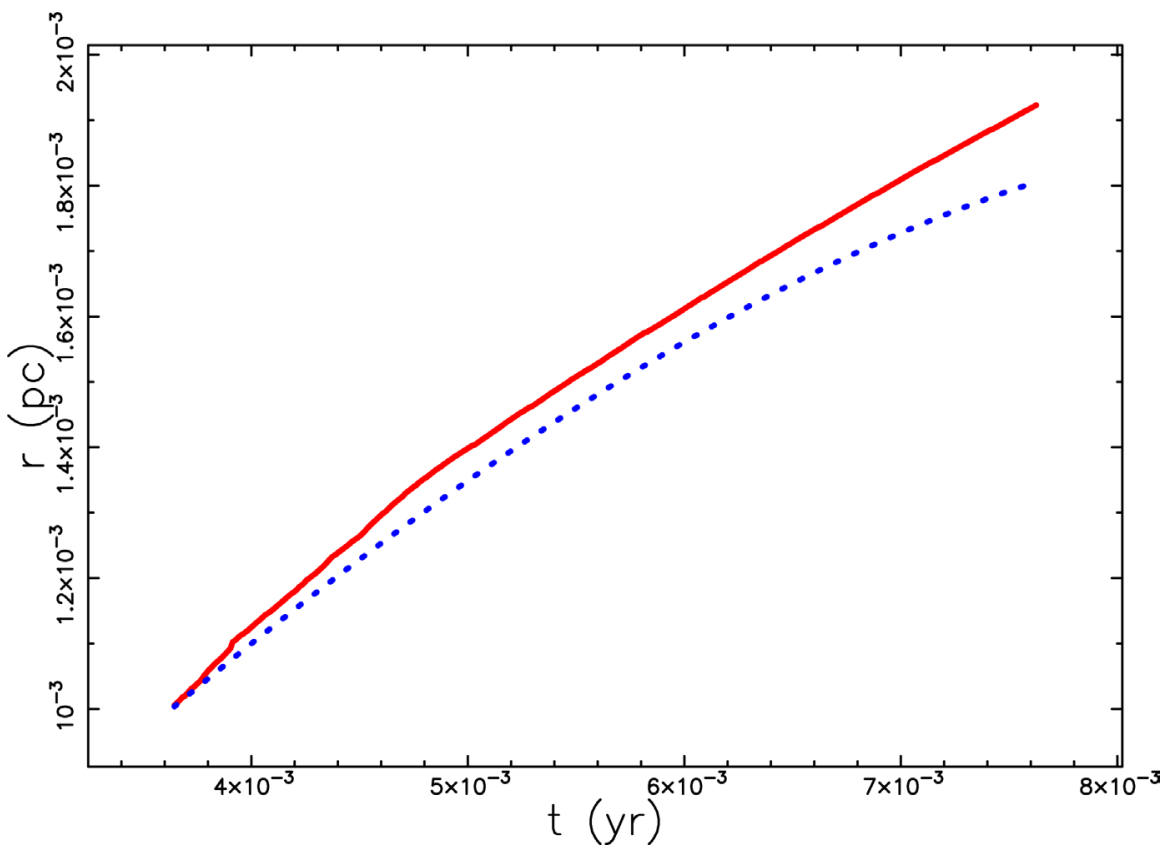

Figure 7. Numerical solution (full-red line) and Taylor solution (blue-dashed line) for the exponential profile, parameters as Figure 6.

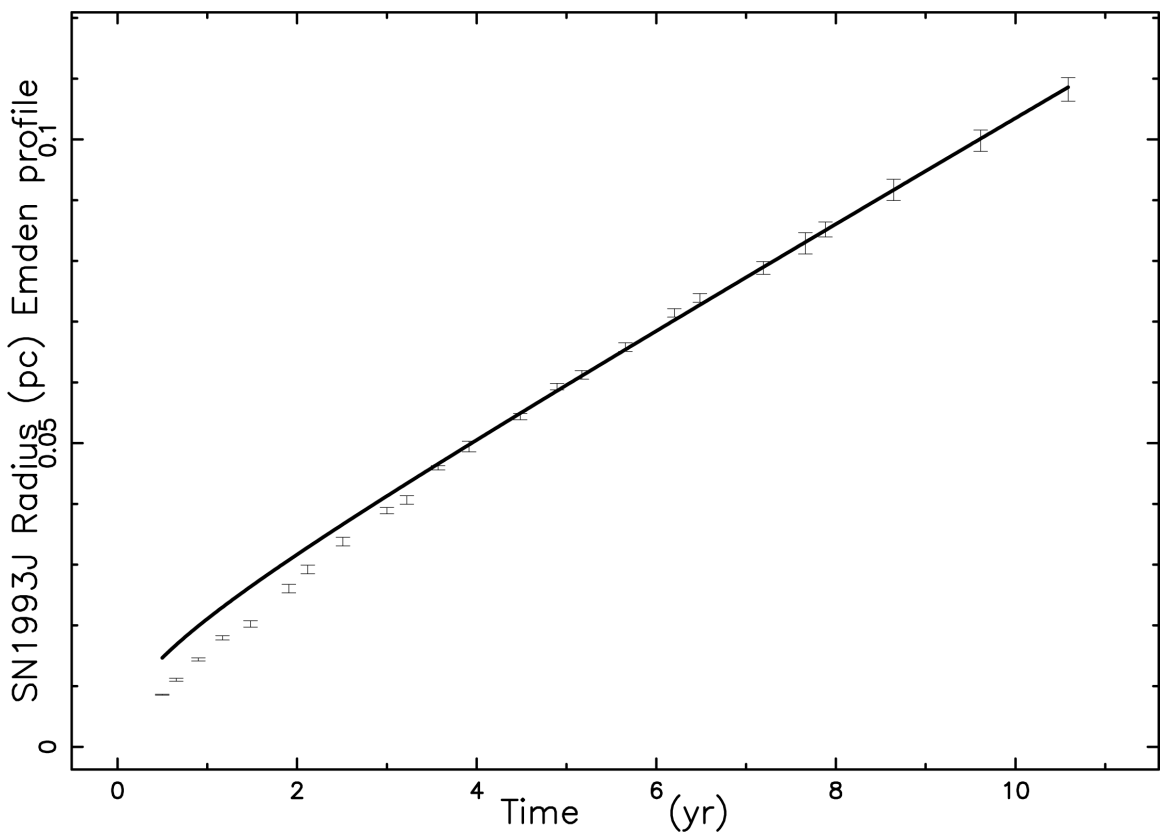

Figure 8. Theoretical radius in the case of an Emden profile (full line) and astronomical data of SN 1993J with vertical error bars. The parameters of the model are $r_{0}=10^{-3} \mathrm{pc}$, $t_{0}=3.6 \times 10^{-3} \mathrm{yr}, \quad \beta_{0}=0.9$ and $b=8.6 \times 10^{-3} \mathrm{pc}$ which gives $\chi^{2}=8888$.

In the case of an Emden profile for density, we present a numerical solution as given by the numerical integration of the differential Equation (26). Figure 8 displays the theoretical model.

Figure 9 presents the Taylor approximation of the trajectory as given by (29) in the restricted range of time $\left[10^{-3} \mathrm{yr}-2 \times 10^{-2} \mathrm{yr}\right]$. 


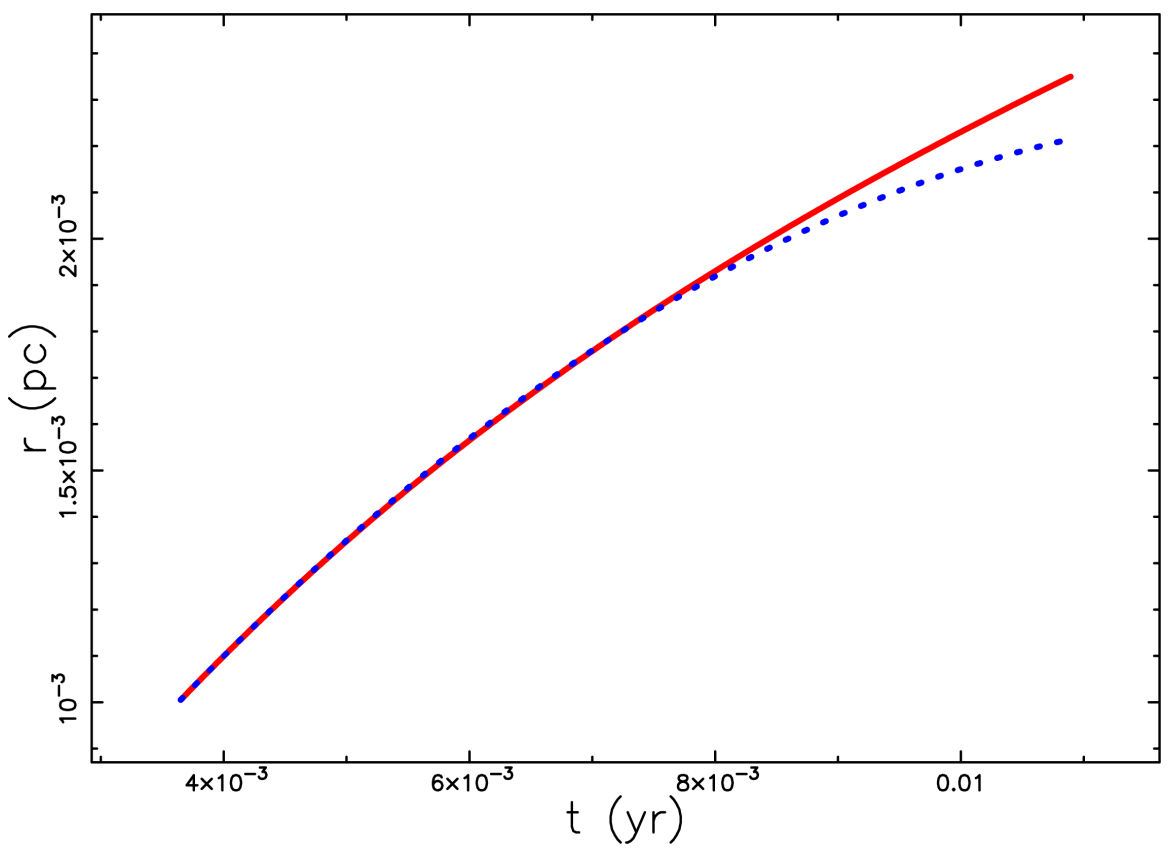

Figure 9. Numerical solution (full-red line) and Taylor solution (blue-dashed line) for the Emden profile, the parameters are the same as in Figure 8.

\section{Sparse Effects}

\subsection{Time Dilation}

For an observer who moves on the expanding shell, the proper time $\tau^{*}$ is

$$
\tau^{*}=\int_{t_{0}}^{t} \frac{\mathrm{d} t}{\gamma}=\int_{t_{0}}^{t} \sqrt{1-\beta^{2}} \mathrm{~d} t,
$$

see [19]. Let us take the example of an Emden profile with the initial trajectory characterized by the Taylor expansion given by Equation (29). The value of $\beta$ as given by the Taylor expansion is

$$
\beta\left(t ; r_{0}, \beta_{0}, t_{0}, b\right)=\frac{1}{c} \frac{\mathrm{d}}{\mathrm{d} t} r\left(t ; t_{0}, r_{0}, v_{0}, b\right)=\frac{B E N}{\left(3 b^{2}+r_{0}^{2}\right)^{5 / 2} \sqrt{-\beta_{0}^{2}+1} r_{0}},
$$

where

$$
\begin{aligned}
B E N= & 9 \sqrt{-\beta_{0}^{2}+1} r_{0}\left(b^{2}+1 / 3 r_{0}^{2}\right)^{2} \beta_{0} \sqrt{3 b^{2}+r_{0}^{2}} \\
& +27 b^{5} c \sqrt{3}\left(\beta_{0}-1\right)\left(\beta_{0}+1\right)\left(\beta_{0}^{2}+\sqrt{-\beta_{0}^{2}+1}-1\right)\left(t-t_{0}\right) .
\end{aligned}
$$

The time dilation can be evaluated once the following integral is done

$$
F\left(t ; r_{0}, \beta_{0}, t_{0}, b\right)=\int \sqrt{1-\beta\left(t ; r_{0}, \beta_{0}, t_{0}, b\right)^{2}} \mathrm{~d} t,
$$

which is

$$
F\left(t ; r_{0}, \beta_{0}, t_{0}, b\right)=\frac{F A^{3} \sqrt{3}}{F B}+t,
$$

where 


$$
\begin{aligned}
F A= & -27 \frac{\left(-\beta_{0} c+c\right)\left(\beta_{0} c+c\right)\left(c \sqrt{-\beta_{0}^{2} c^{2}+c^{2}}-c^{2}+\beta_{0}^{2} c^{2}\right) \sqrt{3} b^{5} t}{\left(3 b^{2}+r_{0}^{2}\right)^{5 / 2} \sqrt{-\beta_{0}^{2} c^{2}+c^{2}} r_{0} c}+\beta_{0} c \\
& +27 \frac{\left(-\beta_{0} c+c\right)\left(\beta_{0} c+c\right)\left(c \sqrt{-\beta_{0}^{2} c^{2}+c^{2}}-c^{2}+\beta_{0}^{2} c^{2}\right) \sqrt{3} b^{5} t_{0}}{\left(3 b^{2}+r_{0}^{2}\right)^{5 / 2} \sqrt{-\beta_{0}^{2} c^{2}+c^{2}} r_{0} c}
\end{aligned}
$$

and

$$
F B=-243 \frac{c^{4} b^{5}\left(\beta_{0}-1\right)\left(\beta_{0}+1\right)\left(\beta_{0}^{2}+\sqrt{-\beta_{0}^{2}+1}-1\right)}{\left(3 b^{2}+r_{0}^{2}\right)^{5 / 2} \sqrt{-\beta_{0}^{2}+1} r_{0}} .
$$

The time dilation is therefore

$$
\tau^{*}=F\left(t ; r_{0}, \beta_{0}, t_{0}, b\right)-F\left(t_{0} ; r_{0}, \beta_{0}, t_{0}, b\right) .
$$

A measure of the time dilation is

$$
D=\frac{\tau^{*}}{t-t_{0}}
$$

with $0<D<1$. The time dilation is displayed as function of the time in Figure 10 and as a function of time and scaling in Figure 11.

\subsection{Radioactivity}

The decay of a radioactive isotope is modeled by the following law for particles, which are in the laboratory frame

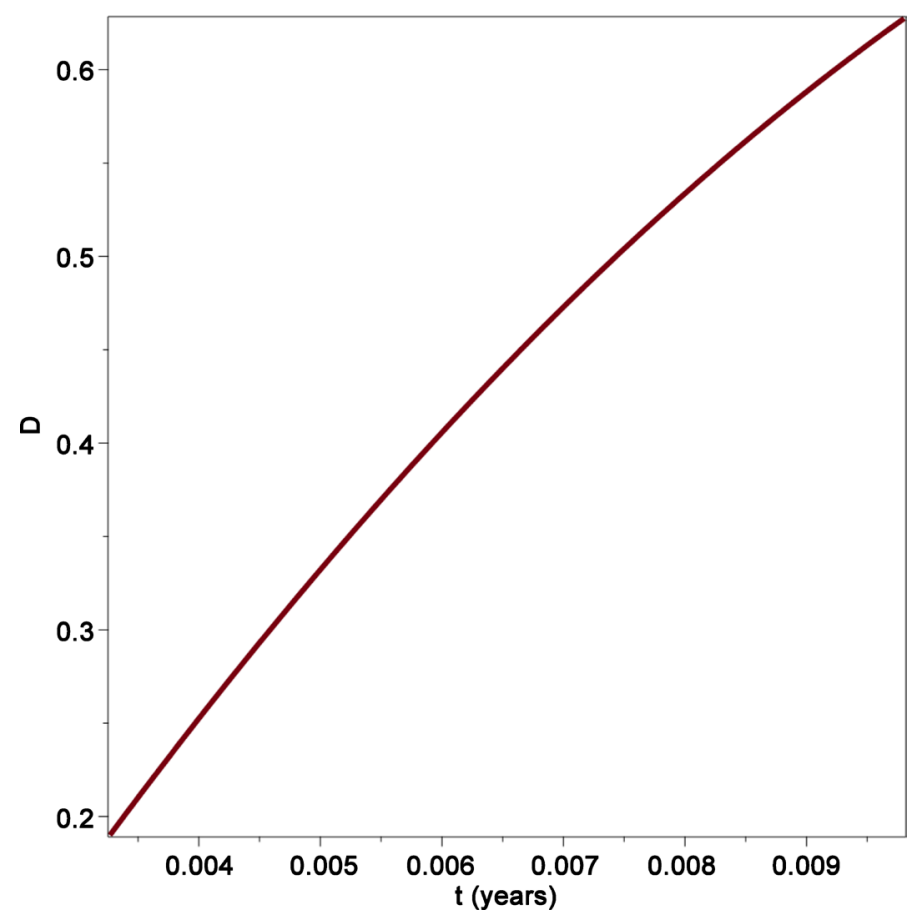

Figure 10. Time dilation as represented by $D$ as a function of time (in years) for the Emden profile, the parameters are the same as in Figure 8. 


$$
N(t)=N_{0} \mathrm{e}^{-\frac{t-t_{0}}{\tau}}
$$

where $\tau$ is the proper lifetime, $N_{0}$ is the number of nuclei at $t=t_{0}$ and the half-life is $T_{1 / 2}=\ln (2) \tau$. In a frame that is moving with the shell, the decay law is

$$
N(t)=N_{0} \mathrm{e}^{-\frac{\tau^{*}}{\tau}}
$$

Let us analyze the isotope ${ }^{56} \mathrm{Ni}$ for which $\tau=8.757 \mathrm{~d}$ or $T_{1 / 2}=6.07 \mathrm{~d}$. We now express the proper lifetime in $\mathrm{yr}(1 \mathrm{yr}=365.24219 \mathrm{~d}), \quad \tau=0.024$ and Table 1 reports the number of nuclei that have survived at a given time. From the above table, it is evident that the number of nuclei that are embedded in the moving layer is bigger when the time dilation is considered.

\section{Conclusions}

The kinetic energy conservation for an expansion in the framework of the thin layer approximation has been extended to SR. We analyzed four types of CSM and we derived the equation for the numerical trajectory. A Taylor expansion for the trajectory has been derived in each of the cases that are modeled by constant, power law, exponential and Emden profile. The numerical results were applied to the real data of SN 1993J. The best results are obtained for a power law

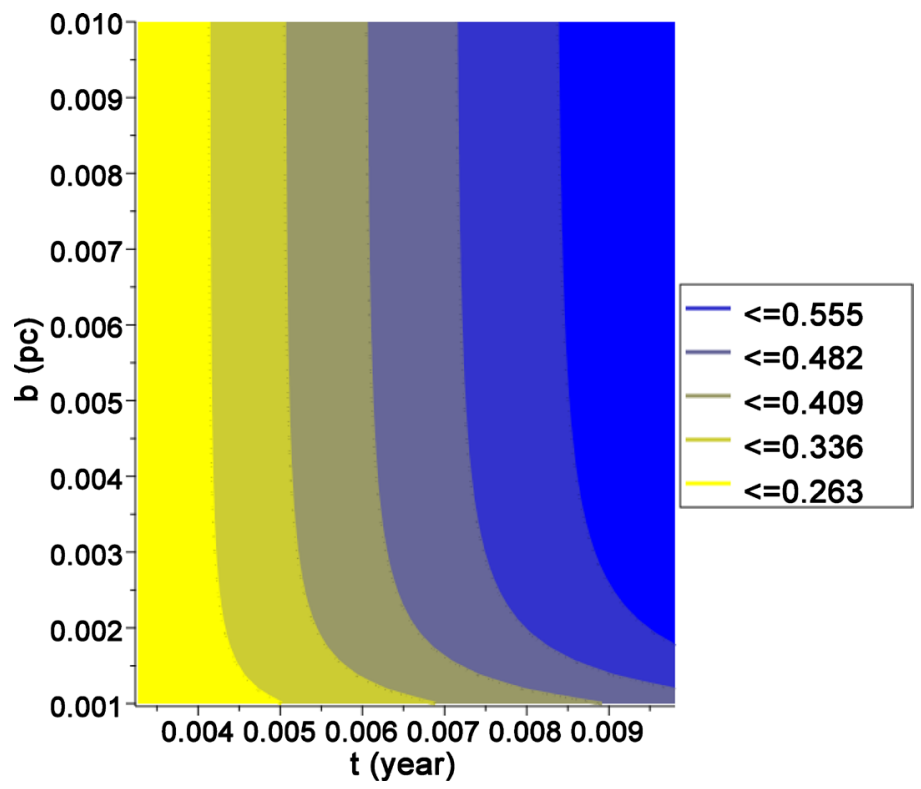

Figure 11. Map of time dilation, $D$, as a function of time (in years) and the scaling $b$ for the Emden profile, the parameters are the same as in Figure 8.

Table 1. Parameters of the radioactive decay for the isotope ${ }^{56} \mathrm{Ni}$ when $t=0.006535 \mathrm{yr}$ and the other parameters are the same as in Figure 8.

\begin{tabular}{ccc}
\hline parameter & no time dilation & time dilation \\
\hline$N$ & $N=N_{0} \times 0.8727$ & $N=N_{0} \times 0.9415$
\end{tabular}


dependence of the CSM with $\alpha=2.15$. The case of an expansion in a medium with constant density is not compatible with the data of SN 1993J. Some evaluations of time dilation and of radioactivity in the early phase of expansion have been done using the Taylor expansion for the trajectory. Here, we processed as astrophysical object only SN 1993j; the connection between SNs and Gamma Ray Bursts is demanded to a forthcoming analysis.

\section{Conflicts of Interest}

The author declares no conflicts of interest regarding the publication of this paper.

\section{References}

[1] Wieland, V., Pohl, M., Niemiec, J., Rafighi, I. and Nishikawa, K.-I. (2016) Nonrelativistic Perpendicular Shocks Modeling Young Supernova Remnants: Nonstationary Dynamics and Particle Acceleration at Forward and Reverse Shocks. The Astrophysical Journal, 820, 62. https://doi.org/10.3847/0004-637X/820/1/62

[2] Bohdan, A., Niemiec, J., Pohl, M. and Matsumoto, Y. (2019) Kinetic Simulations of Nonrelativistic Perpendicular Shocks of Young Supernova Remnants. I. Electron Shock-surfing Acceleration. The Astrophysical Journal, 878, 5. https://doi.org/10.3847/1538-4357/ab1b6d

[3] Bohdan, A., Niemiec, J., Pohl, M. and Matsumoto, Y. (2019) Kinetic Simulations of Nonrelativistic Perpendicular Shocks of Young Supernova Remnants. II. Influence of Shock-Surfing Acceleration on Downstream Electron Spectra. The Astrophysical Journal, 885, 10. https://doi.org/10.3847/1538-4357/ab43cf

[4] Bohdan, A., Pohl, M., Niemiec, J., Vafin, S. and Matsumoto, Y. (2020) Kinetic Simulations of Nonrelativistic Perpendicular Shocks of Young Supernova Remnants. III. Magnetic Reconnection. The Astrophysical Journal, 893, 6. https://doi.org/10.3847/1538-4357/ab7cd6

[5] Taub, A.H. (1948) Relativistic Rankine-Hugoniot Equations. Physical Review, 74, 328-344. https://doi.org/10.1103/PhysRev.74.328

[6] Blandford, R.D. and McKee, C.F. (1976) Fluid Dynamics of Relativistic Blast Waves. Physics of Fluids, 19, 1130. https://doi.org/10.1063/1.861619

[7] Yokosawa, M. (1984) Reverse Shock Wave in Relativistic Explosions. Astrophysics and Space Science, 107, 109-123. https://doi.org/10.1007/BF00649617

[8] Ellison, D.C. and Reynolds, S.P. (1991) Electron Acceleration in a Nonlinear Shock Model with Applications to Supernova Remnants. The Astrophysical Journal, 382, 242. https://doi.org/10.1086/170712

[9] Nakar, E. and Sari, R. (2012) Relativistic Shock Breakouts. A Variety of Gamma-Ray Flares: From Low-luminosity Gamma-Ray Bursts to Type Ia Supernovae The Astrophysical Journal, 747, 88. https://doi.org/10.1088/0004-637X/747/2/88

[10] Ohtani, Y., Suzuki, A. and Shigeyama, T. (2013) Generation of High-Energy Photons at Ultra-Relativistic Shock Breakout in Supernovae. The Astrophysical Journal, 777, 113. https://doi.org/10.1088/0004-637X/777/2/113

[11] Zhao, X.L., Wang, X.F., Maeda, K., et al. (2015) The Silicon and Calcium High-Velocity Features in Type Ia Supernovae from Early to Maximum Phases. The Astrophysical Journal, 220, 20. https://doi.org/10.1088/0067-0049/220/1/20 
[12] Zaninetti, L. (2011) Time-Dependent Models for a Decade of SN 1993J. Astrophysics and Space Science, 333, 99-113. https://doi.org/10.1007/s10509-011-0609-X

[13] Zaninetti, L. (2014) A Classical and a Relativistic Law of Motion for Spherical Supernovae. The Astrophysical Journal, 795, 80.

https://doi.org/10.1088/0004-637X/795/1/80

[14] Marcaide, J.M., Mart-Vidal, I., Alberdi, A. and Pérez-Torres, M.A. (2009) A Decade of SN 1993J: Discovery of Radio Wavelength Effects in the Expansion Rate. Astronomy \& Astrophysics, 505, 927-945.

https://doi.org/10.1051/0004-6361/200912133

[15] Zaninetti, L. (2020) Energy Conservation in the Thin Layer Approximation: I. the Spherical Classic Case for Supernovae Remnants. International Journal of Astronomy and Astrophysics 10, 71. https://doi.org/10.4236/ijaa.2020.102006

[16] Freund, J. (2008) Special Relativity for Beginners: A Textbook for Undergraduates. World Scientific Press, Singapore. https://doi.org/10.1142/6601

[17] Lane, H.J. (1870) On the Theoretical Temperature of the Sun, under the Hypothesis of a Gaseous Mass Maintaining Its Volume by Its Internal Heat, and Depending on the Laws of Gases as Known to Terrestrial Experiment. American Journal of Science, 148, 57-74. https://doi.org/10.2475/ajs.s2-50.148.57

[18] Emden, R. (1907) Gaskugeln: Anwendungen der mechanischen warmetheorie auf kosmologische und meteorologische probleme. B. Teubner, Berlin.

[19] Einstein, A. (1905) Zur Elektrodynamik bewegter Korper. Annalen der Physik, 322, 891-921. https://doi.org/10.1002/andp.19053221004 